\title{
The influence of rewards on incidental memory: more does not mean better
}

\author{
Si Cheng, ${ }^{1,4}$ Ting Jiang, ${ }^{1,4}$ Jingming Xue, ${ }^{1}$ Songxue Wang, ${ }^{1}$ Chuansheng Chen, ${ }^{2}$ \\ and Mingxia Zhang ${ }^{3}$ \\ ${ }^{1}$ Faculty of Psychology, Beijing Normal University, Beijing 100875, China; ${ }^{2}$ Department of Psychological Science, University of California, \\ Irvine, California 92697, USA; ${ }^{3}$ CAS Key Laboratory of Behavioral Science, Institute of Psychology, Beijing 100101, China
}

\begin{abstract}
Studies have revealed that rewards promote long-term memory, even in an incidental way. However, most previous studies using the incidental paradigm have included two reward levels, and it is still not clear how the reward magnitude influences memory. Adopting the incidental paradigm and three reward levels, the current study revealed that the reward magnitude impacted 1-d delayed episodic memory in a nonlinear, inverted U-shaped pattern. An additional experiment showed that there was no reward effect in immediate episodic memory. Our results support the dopaminergic memory consolidation theory and further imply that the reward magnitude needs to be considered in the theory.
\end{abstract}

[Supplemental material is available for this article.]

Recently, studies have revealed that a reward could facilitate memory (Shohamy and Adcock 2010; Miendlarzewska et al. 2016), not just in the intentional paradigm but also in the incidental paradigm. In the intentional paradigm, participants would strategically pay attention to memorize the reward-related items as they are explicitly informed that reward is contingent upon memory performance in a subsequent test when they encode the items (Adcock et al. 2006; Ariel and Castel 2014; Cohen et al. 2014, 2016; Spaniol et al. 2014; Hidi 2016; Miendlarzewska et al. 2016). In the incidental paradigm, the reward accompanies some items during the encoding phase but is independent of memory performance for these items in the test phase (Mather and Sutherland 2011; Murayama and Kitagami 2014). For example, some studies used the category of the items (e.g., living or nonliving objects) to signal rewards in the encoding phase (e.g., participants would anticipate a reward when they met a particular category) and observed a better memory for the reward-related category in the subsequent test (Wittmann et al. 2005; 2011; Patil et al. 2017). In this paradigm, participants always do not know the subsequent memory test before they process it; thus, the enhancement effect on the memory process is explained in terms of pure reward effect rather than top-down attention and effort.

The reward effect on memory is associated with an increased dopamine release that improves the plasticity of the hippocampal memory system (Bromberg-Martin et al. 2010; Chiew et al. 2016). The dopaminergic modulation of the hippocampus could last a long time after reward events occur (e.g., in the memory consolidation phase) (Bayer and Glimcher 2005; Lisman and Grace 2005; Shohamy and Adcock 2010; Flagel et al. 2011; Gruber et al. 2016; Murty et al. 2017), and, correspondingly, behavioral evidence showed reward-related memory improvements in the delayed memory test but not in the immediate memory test (McClure et al. 2004; Murayama and Kitagami 2014; Patil et al. 2017).

Previous studies using the intentional paradigm have revealed that reward values affected memory in a linear way (Castel et al. 2007, 2013); the greater the reward, the better the memory. However, most previous studies using the incidental paradigm re-

${ }^{4}$ These authors contributed equally to this work.

Corresponding author: zhangmx@psych.ac.cn

Article is online at http://www.learnmem.org/cgi/doi/10.1101//m.051722.120. vealed the reward's positive effect on memory by comparing items for two reward levels. It is still unclear how multiple levels of reward influence incidental memory. As many animal studies have revealed that the excitatory responses of midbrain dopaminergic neurons increase as the reward magnitude (or probability) increases (Fiorillo et al. 2003; Satoh et al. 2003; Matsumoto and Hikosaka 2009), one might expect that the impact of the dopaminergic modulation on the hippocampus as well as the reward magnitude on episodic memory occur in a simple linear (or on-off) manner. That is, we are more sensitive to a higher reward, and a higher magnitude would never lead to a worse performance than a lower reward.

However, many studies have revealed that reward magnitude modulates performance in a more complex and nonlinear way. For example, previous studies revealed that reward magnitude influenced the performance of some tasks (e.g., motor learning, intelligence tests) (Kuhbandner et al. 2016; Lee et al. 2019) in an inverted U shape (Mobbs et al. 2009; Aarts et al. 2014; Lee and Grafton 2015). That is, a high reward leads to a less-than-optimal performance compared with a medium reward. Some researchers described this phenomenon as "overmotivation" or "overarousal" and suggested that performance was superior at the level of arousal and dopamine release (Dodson 1915) and that a high reward induced excessive arousal or "overdosed" the dopaminergic system beyond this level and led to a decrement in performance (Mobbs et al. 2009; Aarts et al. 2014). Other researchers have suggested that the negative effect of a high reward was due to the shift of attention and executive control to the internal state (e.g., anxiety) (Callan and Schweighofer 2008; Ariely et al. 2009; Capa and Bouquet 2018; Watanabe et al. 2019) or details of the task (Beilock and Carr 2005; Beilock and DeCaro 2007; Vealey et al. 2014; Lee and Grafton 2015; Lee et al. 2019) that led to a reduction in goal-directed attentional control for the target task. Thus, the negative effect would not occur in tasks that do not require a

(C) 2020 Cheng et al. This article is distributed exclusively by Cold Spring Harbor Laboratory Press for the first 12 months after the full-issue publication date (see http://learnmem.cshlp.org/site/misc/terms.xhtml). After 12 months, it is available under a Creative Commons License (Attribution-NonCommercial 4.0 International), as described at http://creativecommons.org/licenses/by-nc/ $4.0 \%$. 
substantial amount of top-down strategy (e.g., an incidental motor learning task) (Lee et al. 2019).

The inconsistent idea of the relationship between reward magnitude and performance motivated us to explore how reward magnitude influenced incidental episodic memory. Two experiments were conducted in the current study. Experiment 1 comprised an incidental encoding task and a subsequent surprise recognition test $1 \mathrm{~d}$ later to investigate the effect on delayed memory. Sixty healthy participants were randomly assigned to the control and reward groups. One participant was excluded for having memory performance more than three standard deviations below average, resulting in a total sample size of 30 (13 males; mean \pm $\mathrm{SD}=22.4 \pm 3.02 \mathrm{yr}$ ) in the reward group and 29 (13 males; mean \pm $\mathrm{SD}=21.3 \pm 2.36 \mathrm{yr}$ ) in the control group.

In the encoding phase (Fig. 1A), a central fixation cross "+" was presented for $500 \mathrm{msec}$. Then, two images were displayed simultaneously on the left and right sides of the central fixation cross for $1500 \mathrm{msec}$, participants were instructed to judge the number of target images (living images or nonliving images, counterbalanced across participants in each group) in the two images within the following $1500 \mathrm{msec}$ when a "?" was displayed. Informative feedback was provided for $1500 \mathrm{msec}$ after the judgment. To prevent carryover effects (Anderson et al. 2006), participants performed three flanker tasks in which they judged the direction of the middle arrow of a set of three arrows.

Participants in the control and reward groups went through the same encoding procedure, except that they received different feedback for their judgment of the number of target images. A correct response was followed by neutral feedback in the control group and monetary reward feedback based on the number of target images in the reward group. That is, participants in the reward group received no reward $(¥ 0)$, medium reward $(¥ 1 \approx 0.144$ USD) and high reward (¥2) for correct judgments in nontarget trials, onetarget trials and two-target trials respectively. The encoding phase included 20 nontarget (nonreward) trials, 40 one-target (mediumreward) trials, and 20 two-target (high-reward) trials. This design produced four types of images, each including 40 images (160 im- ages in total): nontarget images in the nontarget trials (nonreward images in the nonreward trials), nontarget images in the one-target trials (nonreward images in the medium-reward trials), target images in the one-target trials (reward images in the medium-reward trials), and target images in the two-target trials (reward images in the high-reward trials).

Participants returned 1-d later (the mean interval between the encoding phase and the memory test was $23.73 \mathrm{~h}, \mathrm{SD}=1.51$, in the reward group and was $24.00 \mathrm{~h}, \mathrm{SD}=1.44$, in the control group) for a surprise recognition memory test (Fig. 1B) in which the 160 previously presented images and 160 new images were presented one by one. Participants were instructed to judge whether the images were old or new within $3000 \mathrm{msec}$ and to rate their confidence within $3000 \mathrm{msec}$. Additional procedural details are in the Supplemental Material.

The analyses on participants' response during the encoding phase were in the Supplemental Material and we focused on how reward magnitude influenced memory performance in the main text. We analyzed the memory performance of the participants' correctly judged trials in the encoding phase. The memory performance was indexed by d' score, calculated by the $z$ score of the hit rates (the percentage of "Old" responses for the "Old" stimuli) minus the $\mathrm{z}$ scores of the false alarm rates (the percentage of "Old" responses for the "New" stimuli). The mean hit rates for nontarget images in nontarget trials, nontarget images in one-target trials, target images in one-target trials, and target images in two-target trials were respectively, $0.385,0.416,0.505$, and 0.443 in the reward group and $0.421,0.441,0.434$, and 0.436 in the control group. The mean false alarm rates for nontarget images and target images were respectively, 0.258 and 0.269 in the reward group and 0.304 and 0.335 in the control group.

A 2 (Group: reward vs. control) $\times 4$ (Image Type: nontarget images in nontarget trials vs. nontarget images in one-target trials vs. target images in one-target trials vs. target images in two-target trials) ANOVA with $\mathrm{d}^{\prime}$ score as the dependent variable revealed a significant main effect of Group, $F_{(1,57)}=5.100, P=0.028, \eta^{2}=0.082$, and a nonsignificant main effect of Image Type, $F_{(2.28,129.89)}=$

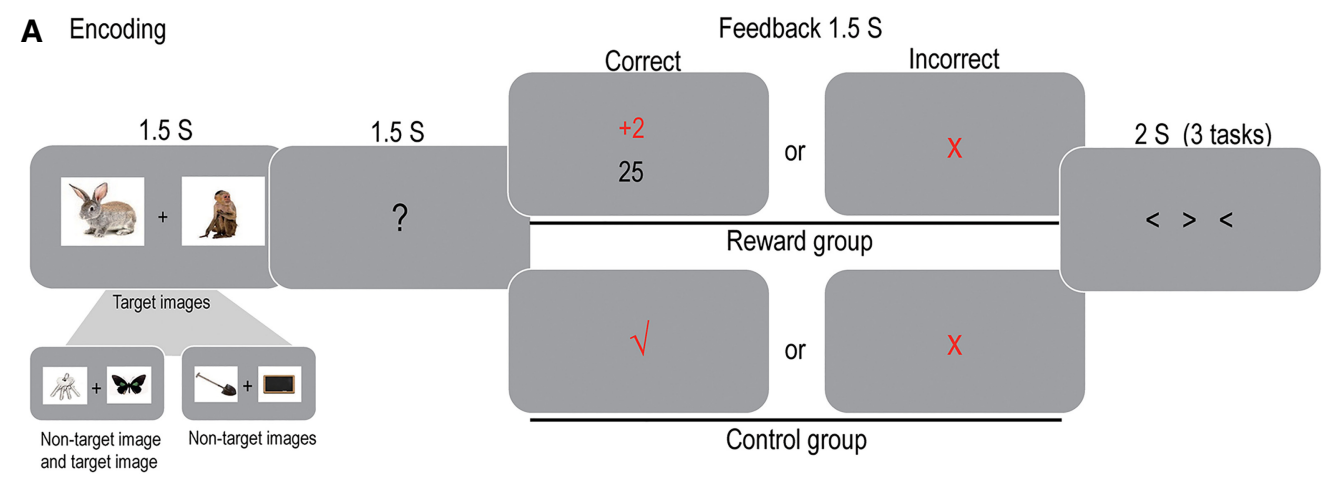

\section{B Recognition Test}

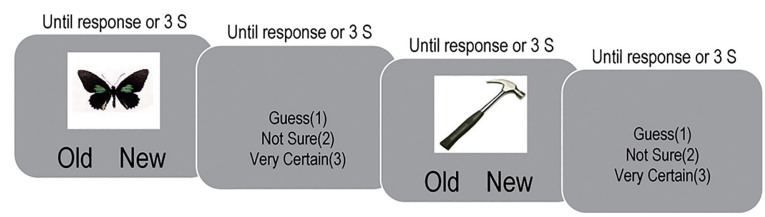

Figure 1. The incidental encoding task and recognition memory test. $(A)$ In the incidental encoding task, participants were instructed to judge the number of target images (living images or nonliving images). In this example, the target images were living images. A correct response was followed by neutral feedback in the control group and monetary reward feedback based on the number of target images in the reward group. (B) $A$ surprise memory test was performed $1 \mathrm{~d}$ later (Experiment 1) or immediately afterward (Experiment 2). Participants were asked to determine whether the images were old or new and to rate their confidence. 


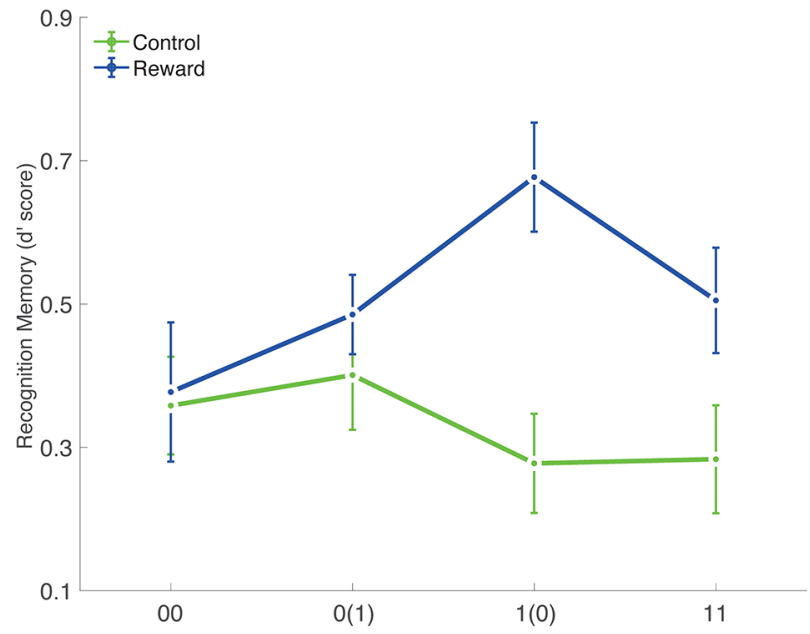

Figure 2. Results from Experiment 1. Recognition memory ( $d^{\prime}$ score) as a function of Group (reward, control) and Image Type $(00=$ nontarget images in the nontarget trials, $0[1]=$ nontarget images in the one-target trials, $1[0]$ $=$ target images in the one-target trials, $11=$ target images in the two-target trials) in the delayed test. Error bars represent the standard error.

$1.519, P=0.220, \eta^{2}=0.026$. The interaction of Group $\times$ Image Type was significant, $F_{(2.28,129.89)}=4.466, P=0.010, \eta^{2}=0.073$ (Fig. 2). Simple main effect analyses revealed that in the control group, there was no significant difference in memory performance across the four types of images, $F_{(3,55)}=0.886, P=0.454$, whereas in the reward group, recognition memory performance was significantly different across the four types of images, $F_{(3,55)}=4.383, P=0.008$. The pairwise comparison analyses revealed that participants in the reward group recognized the reward images in the mediumreward trials significantly better than the nonreward images in the nonreward trials, $t=3.19, P=0.014$, and the reward images in the high-reward trials, $t=2.77, P=0.041$. However, the reward images in the medium-reward trials were not significantly better recognized than the nonreward images in the medium-reward trials, $t=2.23, P=0.165$. The memory performance for the nonreward images in the nonreward and the medium-reward trials and for the reward images in the high-reward trials was not different, $P \mathrm{~s}>0.4$. That is, the reward images in the medium-reward trials were best recognized, and compared with the medium reward, the high reward did not further improve but instead impaired memory performance of reward images. To directly test the reward effect in the four image types, post-hoc $t$-tests at each level between the reward group and the control group were also conducted. The target images in the one-target trials and in the two-target trials were better recognized in the reward group than in the control group, $t=3.87, P<$ 0.001 , and $t=2.11, P=0.040$, whereas the memory performance of nontarget images in the nontarget trials and in the one-target trials in the reward group was not significantly different from that in the control group, $P s>0.3$. It seems the high reward could also promote memory performance though it was not optimal. We also analyzed memory performance with participants' subsequent confidence in their judgments. The overall pattern of the results with confidence was very similar to that without consideration of the confidence, which is presented in the Supplemental Material.

Experiment 1 suggested reward magnitude impacted delayed memory in a nonlinear way. We further tested how reward magnitude affected immediate memory in Experiment 2. Experiment 2 was identical to Experiment 1 except that participants took the recognition test immediately after they finished the encoding task. Sixty participants were recruited and randomly assigned to two groups with one participant excluded for not following the task instructions, resulting in a total sample size of 30 (13 males; mean \pm $\mathrm{SD}=21.3 \pm 2.14 \mathrm{yr}$ ) in the reward group and 29 (13 males; mean \pm $\mathrm{SD}=21.0 \pm 2.24 \mathrm{yr}$ ) in the control group.

Identical to that in Experiment 1, the analyses on participants' response during the encoding phase were in the Supplemental Material and we focused on the reward effect on memory performance in the main text. In the test, the mean hit rates for nontarget images in nontarget trials, nontarget images in one-target trials, target images in one-target trials, and target images in twotarget trials were respectively, $0.549,0.555,0.598$, and 0.601 in the reward group and $0.551,0.549,0.550$, and 0.547 in the control group. The mean false alarm rates for nontarget images and target images were 0.265 and 0.274 in the reward group and 0.287 and 0.236 in the control group. The results in Experiment 2 showed no significant main effect of Group, $F_{(1,57)}=0.342, P=0.561, \eta^{2}=$ 0.006 , or Image Type, $F_{(2.12,120.63)}=2.923, P=0.055, \eta^{2}=0.049$, or interaction of Group $\times$ Image Type, $F_{(2.12,120.63)}=0.016, P=0.987$, $\eta^{2}=0.000$ (Fig. 3). The pattern of the results with confidence was similar to that without consideration of the confidence, which is also presented in the Supplemental Material.

Taken together, our results showed that in the delayed test, the reward images in the medium-reward trials were better recognized than the nonreward images in the nonreward trials and the reward images in the high-reward trials. The memory performance did not differ between the nonreward images in the nonreward and medium-reward trials and the reward images in the high-reward trials. No reward effect on episodic memory was observed in the immediate test. These results demonstrated an inverted $U$ pattern instead of a linear relationship between the reward magnitude and long-term incidental memory.

Many previous studies have revealed that rewards facilitate memory and learning (Shohamy and Adcock 2010; Miendlarzewska et al. 2016). Consistent with these findings, the current study found that reward images (in the medium-reward trials) were better recognized than nonreward images. Some researchers have proposed a dopaminergic memory consolidation theory to explain the reward effect on memory, which suggests that a reward would trigger the release of dopamine that modulates the activity of the hippocampus and other memory-related regions in the consolidation (post-encoding) phase (Wittmann et al. 2005; Murayama and

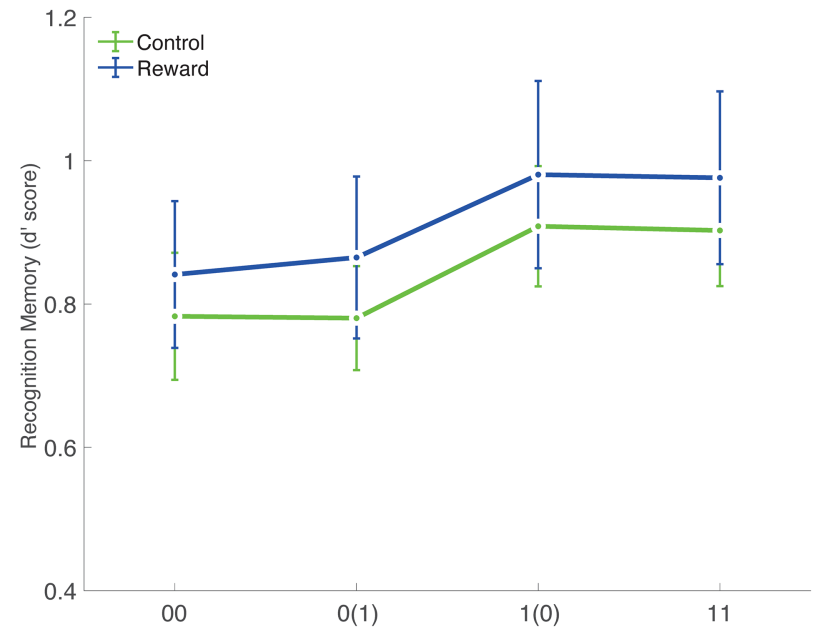

Figure 3. Results from Experiment 2. Recognition memory ( $d^{\prime}$ score) as a function of Group (reward, control) and Image Type $(00=$ nontarget images in the nontarget trials, $0[1]=$ nontarget images in the one-target trials, $1[0]=$ target images in the one-target trials, $11=$ target images in the two-target trials) in the immediate test. Error bars represent the standard error. 
Kitagami 2014; Gruber et al. 2016). Other researchers have suggested that a reward could also impact memory by prompting people to use more effortful strategies on reward-related items (Cohen et al. 2014). Although the current study adopted the incidental paradigm to avoid the use of strategy, one may argue that reward images might automatically capture attention (Anderson et al. 2011; Anderson and Halpern 2017), and be encoded deeper than nonreward images (Murayama and Kitagami 2014), thus inducing an enhancement effect on memory performance. However, it should be noted that the effect only occurred in the delayed test, not in the immediate test, which should be explained by dopaminergic memory consolidation rather than the encoding receiving more attention and cognitive control efforts.

More importantly, the reward images in the high-reward trials were recognized as worse than those in the medium-reward trials. To our knowledge, the current study was the first to reveal high reward impairment in incidental memory. Previous research has shown the negative effect of a high reward on the performance of other tasks (the "choking on money" phenomenon) and proposed two underlying mechanisms to explain the impairment effect of a high reward on performance: excess arousal (and dopamine release) and attention shift (Mobbs et al. 2009; Aarts et al. 2014; Lee and Grafton 2015; Lee et al. 2019). We suggest that in the current study, this effect was due to excess arousal and dopamine release rather than an attention shift to an internal state or to task details because we used an incidental instead of an intentional paradigm to exclude the attention effect. Again, the attention shift could not explain why this effect occurred only in the delayed test, not in the immediate test. One previous study revealed a negative relationship between arousal and intentional spatial memory in reward conditions across trials and participants and suggested arousal as a potential inhibitor in reward-related intentional learning (Murty et al. 2011). Future studies should investigate the physiological and neural bases underlying high reward impairment in incidental memory and learning. Here, we should caution that high rewards "impaired," or had a "negative" effect on memory only when it was compared with the medium reward. When we compared the memory performance between the reward and control groups, we could see the positive effect of reward on the target images for both the one-target (medium-reward) and two-target (high-reward) levels. Thus, the reward could overall enhance memory performance regardless of magnitude and it had an especially positive effect on the medium level.

Although previous studies suggested that dopamine is sent to the hippocampus in a nontargeted way (Adcock et al. 2006; Spaniol et al. 2014; Murty et al. 2017; Pu and Yu 2019) and hence rewards should enhance memory for stimuli nonselectively (Mather and Schoeke 2011; Murayama and Kitagami 2014), we found that the reward in our study only enhanced the memory for the target images, not for the nontarget images in the mediumreward trials. This lack of the reward effect on nontarget images was evident in both the immediate and delayed tests. We speculated that more attention or some kind of arousal-biased competition (Mather and Sutherland 2011) in favor of goal-relevant stimuli (target images) might have interfered with the processing of the simultaneously presented nontarget images and thus attenuated the reward effect on the nontarget images in the immediate test. In addition, a recent study revealed that the effect of dopamine would tag the worthiest items and reprocess them during sleep intensively (Asfestani et al. 2020). It is possible that although dopamine affected the processing of the target and nontarget images nonselectively during the encoding phase, it only tagged and reprocessed the target images during sleep and thus we observed the reward effect on the target images only in the delayed test.

It also should be noted that, although many previous studies revealed the reward effect on the delayed test instead of the imme- diate test, some studies also reported the latter, for both intentional memory (Madan and Spetch 2012; Spaniol et al. 2014; Wolosin et al. 2013) and incidental memory (Murty and Adcock 2014). In the study by Murty and Adcock (2014), the immediate reward effect was observed on the salient items (the novel images that interrupted participant's expectancy) in the reward anticipation stage. It is possible that either the items' salience or the participants' strong expectancy or both might have increased the reward effect in the immediate test. Future studies should investigate the factors that influence the timing of the reward effect.

In addition, the reversed effect of a high reward in the current study was inconsistent with the results of some previous studies that also included more than two levels of reward to test reward and memory (Wittmann et al. 2005; Castel et al. 2007; Callan and Schweighofer 2008; Bunzeck et al. 2010; Wittmann et al. 2011; Madan and Spetch 2012; Castel et al. 2013). These studies used different tasks (or paradigms, or intervals between encoding and test) from those in the current study. For example, previous research showed a linear pattern between reward magnitude and intentional memory in the immediate test (Castel et al. 2007, 2013). Thus, we should caution that reward magnitude may have a complex impact on memory and learning and may depend on many factors, such as task, paradigm, the interval between encoding and test, and personality traits (Callan and Schweighofer 2008).

Finally, we should add a note about the theoretical accounts of current results. In addition to the arousal or dopamine hypothesis aforementioned, our results were also consistent with the "adaptive scaling of reward" theory that suggested the performance was dependent on the relative reward value rather than the absolute reward value (Bunzeck et al. 2010). That is, in our study, the images with a relatively high reward value (the reward images in the medium-reward trials with the relative value of $¥ 1$ ) were better recognized than those with a relatively low reward value (the reward images in the high-reward trials and the nonreward images in the nonreward trials with the relative value of $¥ 0$ ). However, there was a major difference between the study by Bunzeck et al. (2010) study and the current study. Whereas Bunzeck et al. (2010) focused on the effect of the magnitude difference between the expected reward and the actual reward feedback on memory, our study did not lead participants to have an anticipation about the reward before they received the feedback. Future studies should further test the "adaptive scaling of reward" hypothesis in the current task (e.g., to test whether there is the reward effect on the condition of two reward-predicting stimuli, with the cumulative reward magnitude was matched with that of the one-target trials).

In conclusion, this study revealed an optimal effect of a medium reward in long-term incidental episodic memory. This result has two implications for reward and memory research. First, the current study supported dopaminergic memory consolidation. Second, the reward magnitude needs to be considered in efforts to optimize memory and learning.

\section{Acknowledgments}

We thank all people for helpful discussions and comments. We also thank our financial supporters. This research was supported by the National Natural Science Foundation of China (31500 888), the Scientific Foundation of the Institute of Psychology, the Chinese Academy of Sciences (Y4CX122005), and the Open Research Fund of the State Key Laboratory of Cognitive Neuroscience and Learning (CNLYB1507).

\section{References}

Aarts E, Wallace DL, Dang LC, Jagust WJ, Cools R, D'Esposito M. 2014. Dopamine and the cognitive downside of a promised bonus. Psychol Sci 25: 1003-1009. doi:10.1177/0956797613517240 
Adcock RA, Thangavel A, Whitfield-Gabrieli S, Knutson B, Gabrieli JD. 2006. Reward-motivated learning: mesolimbic activation precedes memory formation. Neuron 50: 507-517. doi:10.1016/j.neuron.2006.03.036

Anderson BA, Halpern M. 2017. On the value-dependence of value-driven attentional capture. Atten Percept Psychophys 79: 1001-1011. doi:10 $.3758 / \mathrm{s} 13414-017-1289-6$

Anderson AK, Wais PE, Gabrieli JDE. 2006. Emotion enhances remembrance of neutral events past. Proc Natl Acad Sci 103: 1599-1604. doi:10.1073/ pnas.0506308103

Anderson BA, Laurent PA, Yantis S. 2011. Value-driven attentional capture. Proc Natl Acad Sci 108: 10367-10371. doi:10.1073/pnas.1104047108

Ariel R, Castel AD. 2014. Eyes wide open: enhanced pupil dilation when selectively studying important information. Exp Brain Res 232: 337-344. doi:10.1007/s00221-013-3744-5

Ariely D, Gneezy U, Loewenstein G, Mazar N. 2009. Large stakes and big mistakes. Rev Econom Stud 76: 451-469. doi:10.1111/j.1467-937X.2009 $.00534 . \mathrm{x}$

Asfestani MA, Brechtmann V, Santiago J, Peter A. 2020. Consolidation of reward memory during sleep does not require dopaminergic activation. Cogn Neurosci 32: 1-16. doi:10.1162/jocn_a 01585

Bayer HM, Glimcher PW. 2005. Midbrain dopamine neurons encode a quantitative reward prediction error signal. Neuron 47: 129-141. doi:10 $.1016 /$ j.neuron.2005.05.020

Beilock SL, Carr TH. 2005. When high-powered people fail: working memory and 'choking under pressure' in math. Psychol Sci 16: 101-105. doi:10.1111/j.0956-7976.2005.00789.x

Beilock SL, DeCaro MS. 2007. From poor performance to success under stress: working memory, strategy selection, and mathematical problem solving under pressure. J Exp Psychol Learn Mem Cogn 33: 983-998. doi:10.1037/0278-7393.33.6.983

Bromberg-Martin ES, Matsumoto M, Hikosaka O. 2010. Dopamine in motivational control: rewarding, aversive, and alerting. Neuron 68: 815-834. doi:10.1016/j.neuron.2010.11.022

Bunzeck N, Dayan P, Dolan RJ, Duzel E. 2010. A common mechanism for adaptive scaling of reward and novelty. Hum Brain Mapp 31: 1380-1394. doi:10.1002/hbm.20939

Callan DE, Schweighofer N. 2008. Positive and negative modulation of word learning by reward anticipation. Hum Brain Mapp 29: 237-249. doi:10 $.1002 / \mathrm{hbm} .20383$

Capa RL, Bouquet CA. 2018. Individual differences in reward sensitivity modulate the distinctive effects of conscious and unconscious rewards on executive performance. Front Psychol 9: 148. doi:10.3389/fpsyg.2018 .00148

Castel AD, Farb NA, Craik F. 2007. Memory for general and specific value information in younger and older adults. Mem Cognit 35: 689-700. doi:10.3758/BF03193307

Castel AD, Murayama K, Friedman MC, McGillivray S, Link I. 2013. Selecting valuable information to remember: age-related differences and similarities in self-regulated learning. Psychol Aging 28: 232-242. doi:10 $.1037 / \mathrm{a} 0030678$

Chiew KS, Stanek JK, Adcock RA. 2016. Reward anticipation dynamics during cognitive control and episodic encoding: implications for dopamine. Front Hum Neurosci 10: 555. doi:10.3389/fnhum.2016.00555

Cohen MS, Rissman J, Suthana NA, Castel AD, Knowlton BJ. 2014 Value-based modulation of memory encoding involves strategic engagement of fronto-temporal semantic processing regions. Cogn Affect Behav Neurosci 14: 578-592. doi:10.3758/s13415-014-0275-x

Cohen MS, Rissman J, Suthana NA, Castel AD, Knowlton BJ. 2016. Effects of aging on value-directed modulation of semantic network activity during verbal learning. Neuroimage 125: 1046-1062. doi:10.1016/j.neuroimage .2015.07.079

Dodson JD. 1915. The relation of strength of stimulus to rapidity of habit-formation in the kitten. J Anim Behav 5: 330-336. doi:10.1037/ h0073415

Fiorillo CD, Tobler PN, Schultz W. 2003. Discrete coding of reward probability and uncertainty by dopamine neurons. Science 299: 18981902. doi:10.1126/science.1077349

Flagel SB, Clark JJ, Robinson TE, Mayo L, Czuj A, Willuhn I, Akers CA, Clinton SM, Phillips PE, Akil H. 2011. A selective role for dopamine in stimulus-reward learning. Nature 469: 53-57. doi:10.1038/nature09588

Gruber MJ, Ritchey M, Wang SF, Doss MK, Ranganath C. 2016. Post-learning hippocampal dynamics promote preferential retention of rewarding events. Neuron 89: 1110-1120. doi:10.1016/j.neuron.2016.01.017

Hidi S. 2016. Revisiting the role of rewards in motivation and learning: implications of neuroscientific research. Educ Psychol Rev 28: 61-93. doi:10.1007/s10648-015-9307-5

Kuhbandner C, Aslan A, Emmerdinger K, Murayama K. 2016. Providing extrinsic reward for test performance undermines long-term memory acquisition. Front Psychol 7: 79. doi:10.3389/fpsyg.2016.00079
Lee TG, Grafton ST. 2015. Out of control: diminished prefrontal activity coincides with impaired motor performance due to choking under pressure. Neuroimage 105: 145-155. doi:10.1016/j.neuroimage.2014.10 .058

Lee TG, Acuña DE, Kording KP, Grafton ST. 2019. Limiting motor skill knowledge via incidental training protects against choking under pressure. Psychon Bull Rev 26: 279-290. doi:10.3758/s13423-018-1486-x

Lisman JE, Grace AA. 2005. The hippocampal-VTA loop: controlling the entry of information into long-term memory. Neuron 46: 703-713. doi:10.1016/j.neuron.2005.05.002

Madan CR, Spetch ML. 2012. Is the enhancement of memory due to reward driven by value or salience? Acta Psychol 139: 343-349. doi:10.1016/j .actpsy.2011.12.010

Mather M, Schoeke A. 2011. Positive outcomes enhance incidental learning for both younger and older adults. Front Neurosci 5: 129. doi:10.3389/ fnins.2011.00129

Mather M, Sutherland MR. 2011. Arousal-biased competition in perception and memory. Perspect Psychol Sci 6: 114-133. doi:10.1177/ 1745691611400234

Matsumoto M, Hikosaka O. 2009. Two types of dopamine neuron distinctly convey positive and negtive motivational signals. Nature 459: 837-841. doi:10.1038/nature08028

McClure SM, Laibson DI, Loewenstein G, Cohen JD. 2004. Separate neural systems value immediate and delayed monetary rewards. Science 306: 503-507. doi:10.1126/science.1100907

Miendlarzewska EA, Bavelier D, Schwartz S. 2016. Influence of reward motivation on human declarative memory. Neurosci Biobehav Rev 61: 156-176. doi:10.1016/j.neubiorev.2015.11.015

Mobbs D, Hassabis D, Seymour B, Marchant JL, Weiskopf N, Dolan RJ, Frith CD. 2009. Choking on the money: reward-based performance decrements are associated with midbrain activity. Psychol Sci 20: 955 962. doi:10.1111/j.1467-9280.2009.02399.x

Murayama K, Kitagami S. 2014. Consolidation power of extrinsic rewards: reward cues enhance long-term memory for irrelevant past events. J Exp Psychol Gen 143: 15-20. doi:10.1037/a0031992

Murty VP, Adcock RA. 2014. Enriched encoding: reward motivation organizes cortical networks for hippocampal detection of unexpected events. Cereb Cortex 24: 2160-2168. doi:10.1093/cercor/bht063

Murty VP, LaBar KS, Hamilton DA, Adcock RA. 2011. Is all motivation good for learning? Dissociable influences of approach and avoidance motivation in declarative memory. Learn Mem 18: 712-717. doi:10 $.1101 / \mathrm{lm} .023549 .111$

Murty VP, Tompary A, Adcock RA, Davachi L. 2017. Selectivity in postencoding connectivity with high-level visual cortex is associated with reward-motivated memory. J Neurosci 37: 537-545. doi:10.1523/ JNEUROSCI.4032-15.2016

Patil A, Murty VP, Dunsmoor JE, Phelps EA, Davachi L. 2017. Reward retroactively enhances memory consolidation for related items. Learn Mem 24: 65-69. doi:10.1101/lm.042978.116

Pu M, Yu R. 2019. Post-encoding frontal theta activity predicts incidental memory in the reward context. Neurobiol Learn Mem 158: 14-23. doi:10 $.1016 /$ j.nlm.2019.01.008

Satoh T, Nakai S, Sato T, Kimura M. 2003. Correlated coding of motivation and outcome of decision by dopamine neurons. J Neurosci 23: $9913-$ 9923. doi:10.1523/jneurosci.23-30-09913.2003

Shohamy D, Adcock RA. 2010. Dopamine and adaptive memory. Trends Cogn Sci 14: 464-472. doi:10.1016/j.tics.2010.08.002

Spaniol J, Schain C, Bowen HJ. 2014. Reward-enhanced memory in younger and older adults. J Gerontol B Psychol Sci Soc Sci 69: 730-740. doi:10.1093/ geronb/gbt044

Vealey RS, Low W, Pierce S, Quinones-Paredes D. 2014. Choking in sport: ACT on it!. J Sport Psychol Action 5: 156-169. doi:10.1080/21520704 .2014 .955226

Watanabe N, Bhanji JP, Ohira H, Delgado MR. 2019. Reward-driven arousal impacts preparation to perform a task via amygdala-caudate mechanisms. Cereb Cortex 29: 3010-3022. doi:10.1093/cercor/bhy166

Wittmann BC, Schott BH, Guderian S, Frey JU, Heinze HJ, Düzel E. 2005. Reward-related fMRI activation of dopaminergic midbrain is associated with enhanced hippocampus-dependent long-term memory formation. Neuron 45: 459-467. doi:10.1016/j.neuron.2005.01.010

Wittmann BC, Dolan RJ, Duzel E. 2011. Behavioral specifications of reward-associated long-term memory enhancement in humans. Learn Mem 18: 296-300. doi:10.1101/lm.1996811

Wolosin SM, Zeithamova D, Preston AR. 2013. Reward modulation of hippocampal subfield activation during successful associative encoding and retrieval. JCogn Neurosci 24: 1532-1547. doi:10.1162/jocn_a_00237

Received March 25, 2020; accepted in revised form September 1, 2020. 


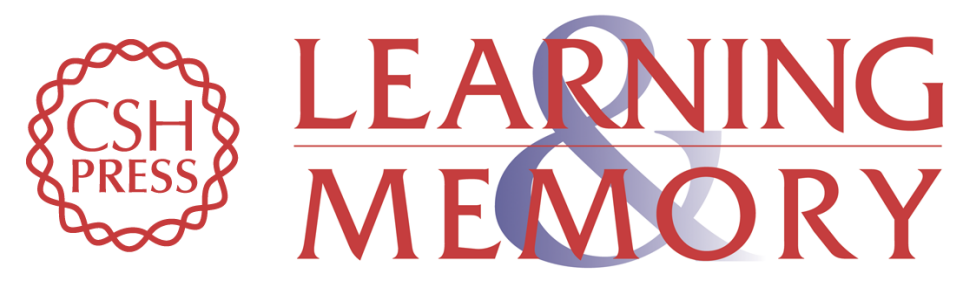

\section{The influence of rewards on incidental memory: more does not mean better}

Si Cheng, Ting Jiang, Jingming Xue, et al.

Learn. Mem. 2020, 27:

Access the most recent version at doi:10.1101/Im.051722.120

\section{Supplemental http://learnmem.cshlp.org/content/suppl/2020/10/13/27.11.462.DC1 Material}

References This article cites 50 articles, 8 of which can be accessed free at: http://learnmem.cshlp.org/content/27/11/462.full.html\#ref-list-1

Creative This article is distributed exclusively by Cold Spring Harbor Laboratory Press for the Commons first 12 months after the full-issue publication date (see

License http://learnmem.cshlp.org/site/misc/terms.xhtml). After 12 months, it is available under a Creative Commons License (Attribution-NonCommercial 4.0 International), as described at http://creativecommons.org/licenses/by-nc/4.0/.

Email Alerting Receive free email alerts when new articles cite this article - sign up in the box at the Service top right corner of the article or click here. 